\title{
Exploring mass variations in the Earth system
}

\author{
Mike Sips, Tobias Rawald, Ingo Sasgen, and Doris Dransch
}

\begin{abstract}
We present a Visual Analytics approach that supports the visual exploration of mass variations in the Earth system in space and time. In close collaboration with geo-scientists, we identified important requirements for approach. Our approach supports scientists to asses the sensitivity of geodetic measurements and to interpret the measured satellite signal for specific processes. Using a combination of three-dimensional spheres, we enable them to compare the differences between simulated mass variations and the measured gravity field. Since these variations change over time, our approach allows scientists to navigate along the temporal scale. In addition, we supply a mechanism to dynamically activate/deactivate multiple components of the simulation model applied. These features enable scientists to better understand the effect of the individual processes on the measured Earth's gravity field. In this paper, we discuss the geo-scientific requirements and how our approach addresses these requirements.
\end{abstract}

Index Terms-Design Study, Measured Data, Simulated Data, Mass Variations

\section{INTRODUCTION}

Satellite missions such as GRACE [6,7] provide high-precision measurements of the Earth's gravity field, which changes from one place to another depending upon the concentration of mass in a region. Mountains usually cause the gravitational force to be higher than it would be on a featureless planet. Conversely, the depression of the landmass through the presence of glaciers million years ago usually causes the gravitational force to be lower. The Earth's gravity field also changes at finer time scales like effects associated with the hydrologic cycle. Analyzing the short-term changes in masses, geo-scientists gain a better understanding of the variability of the Earth's climate system; e.g., melting of ice caps.

Because of Newton's law of gravitation, a variety of masses usually influence the gravity signal at a particular region. The measured signal reflects the impact of several environmental processes, which we refer to as subsystems. This results in two geo-scientific challenges. The first challenge is to develop a better understanding of the measured gravity field. The interpretation of satellite measurements requires Earth system modelers to explore how different masses influence these measurements. This exploration involves the simultaneous comparison of the following four dimensions:

1. The calculated mass variations based on a simulation model.

2. The inferred gravity field changes based on the simulated mass variation.

3. The satellite measurements of the Earth's gravity field changes.

4. The inferred mass variations deduced from the satellite measurements.

The second challenge is to utilize the satellite measurements to improve simulation models capturing the short-term temporal dynamics of mass variations. This challenge requires Earth system modelers to explore the temporal variation of the gravity field and mass changes in different subsystems.

To interpret mass variations, scientists need to explore the impact of different subsystems to a mass variation at a certain position. This poses three Visual Analytics challenges:

- Mike Sips and Ingo Sasgen are with German Research Centre for GeoSciences GFZ. E-mail: \{sips, sasgen\}@gfz-potsdam.de.

- Tobias Rawald and Doris Dransch are with German Research Centre for GeoSciences GFZ and Humboldt University Berlin. E-mail: \{trawald, dransch\}@gfz-potsdam.de.

Manuscript received 31 Mar. 2014; accepted 1 Aug. 2014; date of publication xx xxx 2014; date of current version xx xxx 2014. For information on obtaining reprints of this article, please send e-mail to:tvcg@computer.org.
1. Computation of mass variations over different subsystems

2. Visual encoding of mass variations

3. Exploration of mass variations in space and time

4. Exploration of mass variations in subsystems.

The computation of mass variations over different subsystems needs a fast access to spatial and temporal data of subsystems. We present a hierarchical data model that supports the fast computation of mass variations across different subsystems. Our approach provides four visual views; one for each dimension mentioned above (see Fig. 2). These four views support the geo-scientists to relate mass variations to measured gravitational signals and therefore allow them to attribute mass variations to certain subsystems. Our approach supports the second challenge by providing a slider that allows them to explore mass variations over time. Additionally, Earth system modelers can utilize our approach to validate the geo-scientific algorithms applied to calculate mass variations.

\section{COMPUTATION OF MASS VARIATIONS OVER DIFFERENT SUB- SYSTEMS}

The mass variation data represent independent or coupled physical models of the subsystems in the Earth and satellite measurements. The input data are geo-spatial grids with over one million grid points for each subsystem or measurement. The computation of mass variation over any combination of subsystems poses two requirements on our Visual Analytics approach:

- Fast computation of mass variations

- Integration of additional subsystems in the exploration process

One option is to compute mass variations of many combinations of subsystems in a preprocessing step. This will produce rather large databases which introduce additional performance challenges.

We decided to compute mass variations in main memory on the fly; e.g., mass variation of particular subsystems is has been requested by the user. To enable a fast computation of mass variations, we hierarchically organize the input data according to time, then according to their subsystem and finally according to their spatial grid point (see Figure 1 for an illustration). This hierarchical index structure allows us to efficiently retrieve needed data to calculate mass variations.

To address the second challenge, our system design separates the procedure to read data from a data source from the organization of the data in main memory and the visual interface. The separation provides a flexible abstraction from raw data which allow scientists to integrate additional subsystems. 


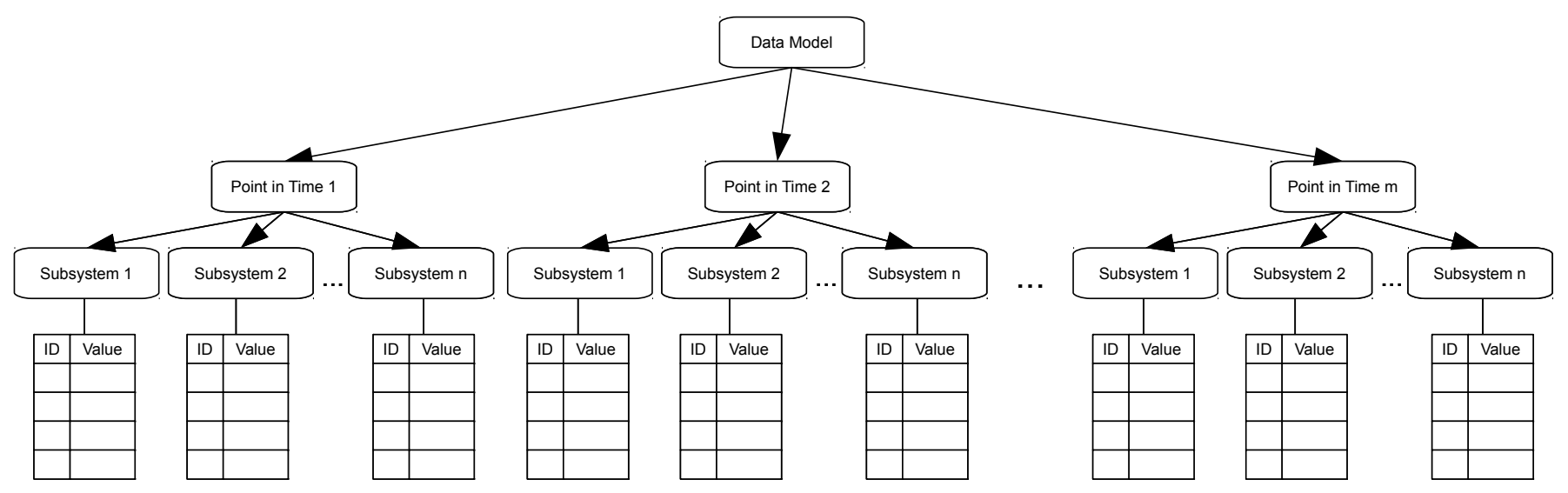

Fig. 1. Hierarchical index structure to support fast in-memory computation of mass variations in space and time, and for combination of subsystems

\section{Visual ENCODING OF MASS VARIATIONS IN SPACE}

The data sets that reflect the mass variations and gravity field changes have specific spatial and temporal resolutions. The spatial resolution specifies the sampling size of the Earth's surface. We refer to a discrete sample of the Earth's surface as grid. The temporal resolution specifies the time steps captured. To address the properties of the data and the scientific challenges, in collaboration with geo-scientists we developed a visualization and interaction concept. In this regard, we defined the following requirements our approach has to comply with:

1. Visualization of mass variation interdependencies,

2. Comparison of different dimensions,

To support these requirements, our interactive visualization interface has two major components. The first component is the main window that provides an overview over all four dimensions (see Fig. 2). For each dimension a three-dimensional sphere visualization is included, depicting data referring to a certain point in time. Our system supports the exploration in space and time by (a) allowing geoscientists to rotate and zoom the spheres and (b) to choose a particular point in time via a slider. Note, the visualized data has the same spatial resolution in each view. To support the exploration of the impact of subsystems to the gravity field, our approach provides a dialog window for each of the four views. The dialog windows present visualization properties and allow the user to adjust the visualization, e.g., select new data by adding or removing subsystems. Each dialog window can be activated or deactivated individually.

In the upcoming sections, we discuss the visualization of the Earth system data, the requirements and the associated tasks in detail.

\subsection{Visualization of mass variation interdependencies}

Since many masses influence the measurement of the gravity field over a region, it is important that the visualization clearly presents these interdependencies. We choose to visualize the grid data using threedimensional spheres. It allows to correlate mass variations on opposite sites of the globe easily.

Visualizing the grid data in three-dimensional space introduces visibility problems; namely, not all parts of a sphere are visible to the user simultaneously. Therefore, our tool provides means of navigation to focus on a region of interest. The user can manipulate the position of the camera, which creates the impression of rotating the sphere along the $X$ and $Y$ axis or to zoom-in and zoom-out. Since an important task is to support scientists to compare variations across multiple dimensions, our system synchronizes the user interaction across the four spheres. The Earth system modeler can interact with the visualization by pressing the arrow keys for rotating the spheres as well as the "+" and the "-" key to zoom-in and zoom-out.

\subsection{Comparison of different dimensions}

Regarding the comparisons of dimensions, we distinguish the following tasks:

- (Ivs. II) Compare the simulated mass variations with the inferred Earth's gravity field changes.

- (III vs. IV) Compare the measured gravity field changes with the inferred mass variations.

- (I vs. IV) Compare the simulated with the inferred mass variations.

- (II vs. III) Compare the inferred with the measured gravity field changes.

We support the comparison of dimensions by arranging the spheres in a special layout; each horizontal and vertical adjacent pair of spheres refers to one of the four tasks (see Fig. 2). Additionally, each view presents further information within a textual overlay, e.g., the type of the dimension displayed.

\section{EXPLORATION OF MASS VARIATIONS IN SPACE AND TIME}

To identify interesting patterns in space and time, the geoscientist has to:

- Navigate through time to identify potentially interesting mass variations.

- Identify and focus on a region with unusual mass variations.

- Distinguish between local peaks and large-area variations.

- Connect the interesting region to its geographical location.

To support the first task, our tool synchronizes the four spheres concerning the temporal navigation. Therefore, all four views present data referring to the same point in time. The user can interactively navigate through time via a slider on the bottom of the main window. Each tick of the slider represents a specific month of a year.

To support scientists to identify interesting mass variations, our tool utilizes a combination of color and size to visually encode mass variations and changes in the gravity field [1]. In the following, we refer to size as the distance between the center of the sphere and a point on its surface.

The challenge in utilizing color as retinal variable is that mass variations and gravity field changes differ in their domain. We address this challenge in our approach by applying different value range intervals for mass variations (I and IV) and gravity field changes (II and III). Note, the color palette applied is the same in all four views. The mapping to color is displayed in the corresponding dialog window for each 


\section{$\Theta 00 \quad$ Mass Variation Explorer}
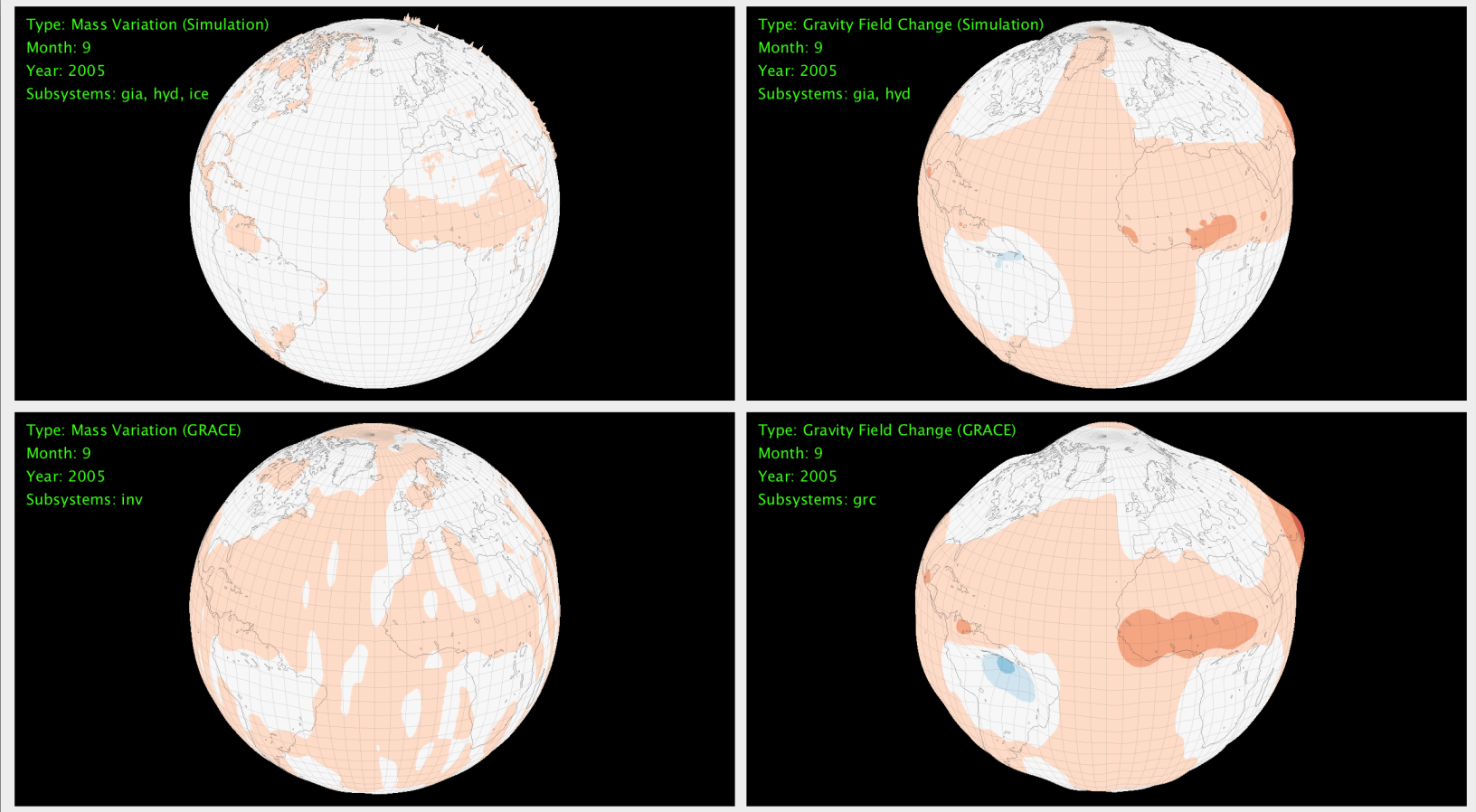

Fig. 2. The main window displays four spherical views; one for each dimension. The roman numerals are not part of the actual visualization but rather serve as a visual aid to identify the dimension displayed. The temporal navigation is realized via a slider at the bottom. Additional information is displayed within a textual overlay. 
dimension. We incorporate the domain knowledge of geo-scientists to create meaningful visual representations of the data. An important convention in the field of Earth system modeling is to use bluish colors to encode negative variations and reddish colors to encode positive changes. To address this convention, we use the color scheme 11class RdBu provided by Color Brewer 2.0 [2]. Note, the center of the scheme is a color close to white, which in our case operates as neutral value, meaning that no variation is present.

To further support the distinction between local peaks and largearea variations, we utilize the second retinal variable size. We enhance the visual encoding by varying the spheres radius for each location based on the associated mass or gravity field variation. Again, we define a default radius that represents the neutral value. A positive variation leads to a radius greater and a negative variation to a radius smaller than the default radius. As a result, the spheres appear distorted. Our color encoding in combination with the distortion supports Earth system modelers to identify interesting mass variations. The mapping to size simplifies the distinction between local peaks and large-area variations. To support effective exploration, the user can switch between two display modes. The first employs only color to encode the variations (Plane), whereas the second combines the encoding using color and size (Elevation).

The fourth important task is to relate unusual mass variations to geographic regions. To support this task, our sphere visualization presents visual landmarks. This comprises a regular grid of prominent longitudes and latitudes as well as the coastlines of continents on the sphere. To distinguish the visual landmarks from the mass variation data, we assign colors that differ from the colors used to visualize the grid data. Additionally, we also adapt the visual landmarks in the display mode Elevation according to the radius computed at any point of the sphere.

\section{EXPLORATION OF MASS VARIATIONS IN SUBSYSTEMS}

This requirement is a central aspect of understanding the impact of a single subsystem to the measured gravity signal. Geo-scientists determine its influence by comparing the intensity of a subsystem to the measured gravity field or the inferred mass variation. Our approach enables to attribute the high gravitational signal in Fig. 2(III) to the hydrological subsystem. The hydrological subsystem shows a high peak at the Amazon River which resembles the measured gravity signal. The activation of other subsystems, e.g., in Fig. 2(II) the subsystem glacial-isostatic adjustment (GIA) is also activated, did not change the simulated gravity signal.

Hence, our tool has to support the following two tasks:

- Activate/deactivate a subsystem signal belonging to the simulated mass change variation dimension.

- Activate/deactivate a subsystem signal belonging to the inferred gravity field change dimension.

The user can activate/deactivate subsystems in the dimension (I), (II). We provide controls to support this analytical step with the dialog window. Each signal has either one of the two states activated or deactivated. Therefore, we supply a binary check-box for each subsystem signal available in the data. After subsystems are activated or deactivated, our tool updates the corresponding sphere visualization automatically.

\section{Related Work}

The visualization and interactive exploration require the integration of measured data and simulated data into a common data model. The simulation data is generated by independent or coupled physical models of the subsystems of the Earth; here, it includes continental hydrology, cryosphere, ocean, and GIA. To support exploratory analysis, our visualization system needs to have efficient access to spatial (grid points) and temporal data (time steps). We developed an efficient data model to enable fast access to the spatial and temporal data. In contrast to [9], we present a thorough task analysis [10] and explain our design decisions in detail.
The comparison of simulated and measured data is an important challenge in many geo-scientific fields. It has stimulated research projects in the visualization community; e.g., [10], to exploit the benefits of interactive visualization for this comparison. Geo-scientists use visualization and exploration tools to extract information and meaning from complex geo-scientific data (see for example [4]). Commercial systems such as Avizio [5] and scientific systems like NCAR Graphics [8] are available to geo-scientists to support exploration of complex geo-scientific data sets. In contrast to these visualization approaches, we present a Visual Analytics approach that combines the fast computation of mass variations and their interactive visual exploration.

\section{Conclusion}

In cooperation with Earth system modelers, we developed an approach that allows to visually explore spatial and temporal mass variations and their impact to the Earth's gravity field. Our approach supports the central task of comparing the measured gravity field with simulated mass variations. Our Visual Analytics approach is based on a thorough task analysis. We regularly met with geo-scientists to identify the spot at which Visual Analytics technology can provide a valuable contribution to the scientific problem. On this basis, we developed requirements for an exploration approach. We iteratively refined these requirements to identify the underlying analytical tasks. Finally, we derived appropriate visual metaphors and interaction mechanisms to support interactive visual analysis and exploration.

Our Visual Analytics approach supports scientists to interpret gravity field measurements. The visual exploration of mass variations and relation to subsystems helped scientists to interpret superimposed satellite signals. The key features of our approach are threedimensional visualizations of mass variations and the gravity field changes. Our tool provides means of navigation in space and time to identify and focus on interesting variations. Geo-scientists use our prototypical exploration tool in multiple scientific scenarios; e.g., to analyze incoming data from current satellite missions. A next step is to integrate our exploration tool in a central system environment for the management of Earth system data [3]. For this purpose, we need to connect the tool to additional databases. Moreover, we plan to extent the visualization methods to support temporal analysis through slicing the spheres; each slice representing a different point in time. Concatenating the slices, temporal navigation is implemented by rotating spheres. This approach would make the slider obsolete.

\section{REFERENCES}

[1] J. Bertin. Semiology of Graphics: Diagrams, Networks, Maps. Esri Press, Redlands, 2011

[2] C. Brewer, M. Harrower, and The Pennsylvania State University. ColorBrewer: Color Advice for Maps, March 2014.

[3] ESA/ESTEC \& The NG2 Team. Assessment of a Next Generation Gravity Mission to Monitor the Variations of Earth's Gravity Field. Technical report, 2011. NG2-ASG-TN-001, Issue 1.

[4] European Geosciences Union General Assembly 2013. Techniques and tools for effective visualization in the geosciences, March 2013.

[5] FEI Visualization Sciences Group. Avizio Visualization Software, March 2014.

[6] Helmholtz Centre Potsdam - GFZ German Research Centre for Geosciences. Gravity Recovery and Climate Experiment (GRACE) Mission, March 2014.

[7] NASA Earth Observatory. Gravity Recovery and Climate Experiment (GRACE), March 2014.

[8] National Center for Atmospheric Research. NCAR Graphics, March 2014.

[9] M. Sips, T. Rawald, I. Sasgen, and A. Unger. Exploration of spatial and temporal signatures in multiple components of a geodetic earth system model. In R. Seppelt, A. Voinov, S. Lange, and D. Bankamp, editors, Managing Resources of a Limited Planet: Pathways and Visions under Uncertainty. 2012.

[10] A. Unger, S. Schulte, V. Klemann, and D. Dransch. A visual analysis concept for the validation of geoscientific simulation models. IEEE Transactions on Visualization and Computer Graphics, 18(12):2216 - 2225, 2012. 